\section{Actualización de Criterios Diagnósticos y Tratamiento de la Angina de Ludwig}

Update of Diagnostic and Treatment of Ludwig's Angina

\begin{abstract}
Resumen
La angina de Ludwig es una celulitis rápidamente progresiva que compromete bilateralmente los espacios submaxilar, sublingual y submentoniano; y que se origina principalmente por infecciones periapicales de las segundas y terceras molares mandibulares. Debido a la rápida progresión del cuadro, que en algunos casos puede incluso poner en peligro la vida del paciente, es sumamente importante conocer los criterios necesarios para realizar un diagnóstico oportuno de la angina de Ludwig, el cual nos permita brindar el tratamiento más adecuado para detener la progresión del cuadro y prevenir la aparición de complicaciones potencialmente mortales.

Palabras claves: Angina de Ludwig, Celulitis Odontogénica, Diagnóstico, Tratamiento, Anaerobios.

\section{Abstract}

Ludwig's angina is a rapidly progressive cellulitis that involves sub mandibular, sub lingual and sub mental spaces bilaterrally. This was due mainly to per apical infections of the second and third mandible molars. Due to the rapid advancement of the clinical (which in certain occasions could even threaten the patient's life) it is extremely important to know clearly the criteria for early diagnosis of Ludwig's angina, which will enable us to provide the most appropriate treatment to halt the progress of the clinical and will prevent the onset of life-threatening complications.
\end{abstract}

Palabras claves: Ludwig's angina, Odontogenic Cellulitis, Diagnostic, Treatment, Anaerobes.
Casos Clínicos

Evelin García León ${ }^{1}$, Eliberto

Ruíz Ramírez ${ }^{1}$, Victoria

Caldas Cueva ${ }^{1}$, Juan

Sánchez Huamaní1, Janet

Tenorio Estrada ${ }^{1}$, Oscar

Barreda Torres', Edwin

Velásquez Inga' ${ }^{1}$, Cristian

Sayán Sánchez', Yuri Castro

Rodríguez', Lisbeth Pérez

Cahuaya ${ }^{1}$, César Franco

Quino', Juan Eche Herrera', Carlos Erazo Paredes ${ }^{1}$, Víctor

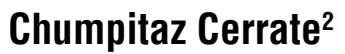

\footnotetext{
${ }^{1}$ Estudiante de Odontología. Facultad de Odontología, Universidad Nacional Mayor de San Marcos.

${ }^{2}$ Profesor Auxiliar del Departamento Académico de Ciencias Básicas de la Facultad de Odontología de la Universidad Nacional Mayor de San Marcos.
}

Correspondencia:

Mg CD Víctor Manuel Chumpitaz Cerrate Facultad de Odontología UNMSM

Departamento Académico de Ciencias Básicas vchumpitazcerrate@hotmail.com

Fecha de recepción: 12-03-10

Fecha de aceptación:12-08-11

\section{Introducción}

La angina de Ludwig es una infección grave y de rápido progreso que se extiende por el piso de boca y que afecta simultáneamente los espacios submaxilar, sublingual y submentoniano. Fue descrita por primera vez en 1836 por Wilhelm Frederick von Ludwig como una induración gangrenosa de tejidos blandos en el cuello y piso de boca. La infección generalmente comienza con un cuadro agudo de celulitis difusa, manifestándose bilateralmente como una tumefacción de consistencia dura. ${ }^{1}$ Su principal origen suele relacionarse a infecciones odontogénicas, sobre todo infección periapical de la segunda y tercera molar inferior (70-80\%), cuyas raíces se extienden debajo de la cresta del músculo milohiodeo de donde se expande la infección hacia otros espa- cios. ${ }^{2,3}$ También se han descrito como causas fractura mandibular, sialoadenitis submandibular, infecciones faríngeas o amigdalinas, infecciones a cuerpo extraño o infecciones secundarias a un carcinoma de células escamosas; también se ha relacionado a la presencia de algunas enfermedades como anemia aplásica, neutropenia, inmunodeficiencias, diabetes o a condiciones como alcoholismo, desnutrición y trasplante de órganos. Los agentes causantes incluyen bacterias aerobias y anaerobias, incluyendo estreptococos y estafilococos, los cuales producen una importante necrosis muscular. ${ }^{4,5}$

El objetivo del presente artículo es realizar una revisión exhaustiva de los criterios diagnósticos que permitan realizar una identificación oportuna de los pacientes con angina de Ludwig; así mismo revisar los protocolos de tratamiento farmacológico y quirúrgico que han demostrado una mayor eficacia en los pacientes con diagnóstico de angina de Ludwig.

\section{Metodología}

Se realizó una revisión de la bibliografía publicada en artículos de revistas especializadas y tesis de postgrado, durante los últimos 10 años, a partir de los cuales se seleccionó la información mejor documentada, más relevante y relacionada específicamente a los criterios necesarios para realizar un adecuado diagnóstico y tratamiento farmacológico y quirúrgico de la angina de Ludwig.

La información obtenida al finalizar la revisión bibliográfica ha sido organizada en tres secciones: (a) criterios diagnósticos, (b) complicaciones, (c) tratamiento farmacológico y no farmacológico. 


\section{Criterios Diagnósticos}

La angina de Ludwig se distingue por una celulitis rápidamente progresiva localizada en el espacio submandibular, que afecta las áreas sublingual y submentoniana y los tejidos blandos del piso de la boca, sin involucrar los ganglios linfáticos. ${ }^{6-7} \mathrm{El}$ examen clínico es de extrema importancia, debido a que en la gran mayoría de casos el diagnóstico es alcanzado basándose en los hallazgos clínicos, para ello debemos seguir los criterios diagnósticos reportados en la literatura como son: celulitis submandibular con proceso gangrenoso y exudado serosanguinolento, que compromete los espacios submandibular, submentoniano y sublingual bilateralmente, pudiendo presentar trismus mandibular, limitación de la apertura bucal, elevación de la lengua, disfagia y odinofagia. ${ }^{6,8-12}$ Además las personas afectadas muestran una respuesta inflamatoria sistémica que se manifiesta con fiebre, taquicardia y taquipnea; así como protrusión de la lengua con elevación del piso de boca e induración blanda a la palpación y dolor cervical anterior. ${ }^{13}$ Se sospecha compromiso del espacio submandibular y la vía aérea, cuando el paciente toma la posición de olfateo para maximizar la entrada de aire a los pulmones, tiene disfonía, estridor, taquipnea, utiliza los músculos accesorios y maneja mal las secreciones. $^{3}$

Como medio auxiliar de diagnóstico y para guiar un drenaje quirúrgico, se deben solicitar exámenes imagenológicos para valorar las estructuras comprometidas. Se puede solicitar radiografías anteroposteriores de perfil de tórax para buscar evidencias de mayores alteraciones. $^{9,14}$ Esta radiografía puede mostrar el ensanchamiento del espacio mediastínico y la existencia de aire a este nivel. Cuando la infección alcanza el mediastino el paciente presenta dolor retroesternal, disnea severa y tos no productiva. También puede presentar edema y crepitación en el tórax superior. En dichos casos el estado general estará claramente alterado por fiebre elevada, escalofríos y postración extrema. La radiografía lateral de cuello es útil para observar un desplazamiento de la pared posterior de la faringe, junto con la presencia de gas libre. ${ }^{2}$ Una tomografía computarizada es importante porque puede facilitar hallazgos como celulitis en los tejidos blandos de los espacios submandibular y sublingual, colecciones fluidas y formación de abscesos; y además facilita la identificación del compromiso de los espacios latero- faríngeos, retrofaríngeos y mediastino, y la visualización de deformidades o dislocamiento de las vías aéreas. ${ }^{14-15}$

También se pueden solicitar las ultrasonografías de los tejidos blandos, por ser una modalidad de examen de imagen ideal para diferenciar infecciones superficiales de infecciones profundas más severas. ${ }^{15}$

\section{Complicaciones}

La angina de Ludwig es potencialmente letal, debido a que puede extenderse rápidamente al espacio sublingual y submandibular. Por extensión posterior, puede diseminarse al espacio parafaríngeo y por vía anterior al mediastino. El edema de la glotis puede obstruir las vías aéreas superiores y puede resultar imposible el habla, presentándose disfagia y disnea intensa. ${ }^{16}$ A la gravedad derivada del cuadro infeccioso que puede traer consigo una sepsis o un shock séptico, se añade el peligro inminente de asfixia además de otras complicaciones como fascitis necrotizante y mediastinitis. ${ }^{17}$ El cuadro de mediastinitis descendente necrotizante es una rara pero letal infección de la fascia cervical con compromiso mediastínico, donde la infección progresa a través de los planos faciales cervicales con celulitis, necrosis y formación de abscesos. Cuando la infección alcanza el mediastino el paciente presenta dolor retroesternal, disnea severa y tos no productiva, también puede presentar edema y crepitación en el tórax superior. La mediastinitis presenta una tasa de mortalidad del $67 \%$, para disminuir dicha tasa es necesario un tratamiento quirúrgico. ${ }^{18,19}$

La fascitis necrotizante aguda se caracteriza por grandes necrosis y formación de gas situados en la zona del tejido celular subcutáneo y en la fascia superficial. Es una afectación de los músculos y la piel, dando lugar a una mionecrosis y manchas en la zona que vienen dadas por la trombosis de los vasos alimentarios que pasan a través de las fascias infectadas. El dolor puede ser intenso inicialmente, mientras que en su evolución puede presentarse parestesia o anestesia en la zona debido al compromiso nervioso. Si el proceso necrotizante continúa extendiéndose, compromete a los tejidos adyacentes, apareciendo complicaciones locales o sistémicas. Existe una afectación del estado general con fiebre, crepitación y manifestaciones sistémicas de la sepsis. ${ }^{6}$

\section{Tratamiento Farmacológico y No Far- macológico}

El objetivo principal del tratamiento de la angina de Ludwig es dar soporte a la vía aérea y erradicar el proceso infeccioso mediante la utilización de fármacos y medidas quirúrgicas. Para el tratamiento podemos considerar tres aspectos básicos: soporte de la vía aérea, tratamiento farmacológico y procedimientos quirúrgicos.

\section{Soporte de la vía aérea}

El compromiso de la vía aérea se puede presentar hasta en un tercio de los pacientes, y el manejo se debe realizar en aquellos que demuestren edema submandibular con elevación y protrusión de la lengua, estridor, dificultad en el manejo de secreciones, ansiedad y cianosis. ${ }^{20}$ Para mantener la vía aérea se puede aplicar un fármaco como la dexametasona $4 \mathrm{mg} \mathrm{c} / 6$ horas durante 2 días, ${ }^{3}$ o cuando no es suficiente utilizar un método más invasivo como la intubación nasotraqueal con visión de fibra óptica. No se recomienda intubación a ciegas por el peligro de broncoaspiración que se puede ocasionar al desgarrar la mucosa. El edema del piso de boca y el trismus generalmente producen dificultades para la intubación, en esos casos se pueden realizar una traqueotomía o cricotiroidectomía. La intubación debe realizarse sin narcóticos, los cuales pueden exacerbar la dificultad respiratoria. ${ }^{19}$

\section{Tratamiento Farmacológico}

Para elegir adecuadamente el tratamiento antibiótico debemos conocer a las bacterias más predominantes en la angina de Ludwig. Las bacterias anaerobias más aisladas son Prevotella, Porphyromonas, Fusobacterium y Peptostreptococos spp; y las bacterias aerobias son Estreptococos del grupo A, Estreptococos viridans, Staphylococcus aureus y Haemophilus influenzae.?

Para el tratamiento antibiótico de la angina de Ludwig la mayoría de autores recomiendan:

Adultos: Penicilina G 4 a 30 millones de UI al día por vía IV divididas cada 4 o 6 horas o por infusión intravenosa continua; asociada a Metronidazol $1 \mathrm{~g}$ de carga seguido de $500 \mathrm{mg}$ cada 6 horas por vía IV, aunque otros autores recomiendan $1 \mathrm{~g}$ cada 12 horas. $^{2}$

Penicilina G cristalina asociada a Clindamicina; Penicilina - Clindamicina - Amikacina; y Ceftazidima asociada a Clindamicina. ${ }^{21}$; Ampicilina con Sulbactam por via IV. ${ }^{6}$ 
Penicilina con Metronidazol o Penicilina con Clindamicina. ${ }^{6}$; Otras alternativas para combatir los microorganismos productores de betalactamasas son: Ampicilina/sulbactam, piperacilina/ tazobactam, amoxicilina/clavulanato y cefotetán o carbapenems. ${ }^{6}$; Fluorquinolona con metronidazol; clindamicina o cefoxitina. ${ }^{22}$

Ampicilina, clindamicina y vancomicina. ${ }^{23}$; Algunos estudios asocian también al tratamiento farmacológico: Gentamicina por vía IV, ${ }^{2}$ Amikacina por vía IV o Cefotaxima por vía IV. ${ }^{24}$

Nińos: Clindamicina $(30$ a $40 \mathrm{mg} / \mathrm{kg} /$ dia) IV o IM..$^{25}$ Ceftazidima $(150 \mathrm{mg} /$ $\mathrm{kg} /$ dosis) IV. ${ }^{25}$

Amoxicilina asociado a acido clavulanico de 40 a $50 \mathrm{mg} / \mathrm{kg} / \mathrm{día}$ IV. ${ }^{26}$ Penicilina $\mathrm{G}$ sódica cristalina, en pacientes menores de $27 \mathrm{~kg}$ de peso la dosis utilizada es de 50,000 UI $/ \mathrm{kg} /$ día cada 6 horas por 7 a 10 días IV. $^{26}$ Ampicilina/sulbactam $650 \mathrm{mg}$ via IV, cloxacilina $250 \mathrm{mg}$ IV qid y metronidazol $80 \mathrm{mg}$ via IV.27 Otras alternativas: aminoglucósidos como gentamicina, oxacilina, cefazolina, cloranfenicol, estreptomicina. ${ }^{28}$

\section{Tratamiento quirúrgico}

El tratamiento quirúrgico de las infecciones que afectan a los espacios faciales, como la angina de Ludwig, está indicado en las siguientes circunstancias:

1) Diagnóstico de celulitis en dos o más de los espacios faciales de cabeza y cuello.

2) Signos clínicos significativos de infección.

3) Infecciones en espacios que pueden comprometer la vía aérea o sean susceptibles de complicaciones.

La descompresión quirúrgica está indicada en abscesos de gran tamaño o si después de 24 a 48 horas de antibióticos parenterales no se observa mejoría. En abscesos pequeños la aspiración con aguja es una alternativa al drenaje quirúrgico con antibióticos. ${ }^{29}$

Para realizar la descompresión quirúrgica, se debe considerar las zonas anatómicas comprometidas: el espacio submandibular con una incisión de 0.5 a 5 $\mathrm{cm}$ inferior al ángulo de la mandíbula, y luego se realiza la disección para explorar los espacios; el espacio submental con una incisión horizontal de 0.5 a $2 \mathrm{~cm}$ paralela al borde inferior de la sínfisis de la mandíbula; el espacio sublingual se realiza por vía intraoral con incisiones pequeñas de $0.5 \mathrm{a} 2 \mathrm{~cm}{ }^{30}$
Por último, debe atacarse la fuente de la infección, la cual en la mayoría de los casos es una pieza dentaria cariada y cavitada. La extracción temprana del diente comprometido ha mostrado disminuir el tiempo de recuperación y disminuir la necesidad de incisión y drenaje. $^{29}$

El drenaje se realiza en quirófano con anestesia general e intubación endotraqueal. Se realiza una incisión amplia submentoniana o sublingual, dejando drenaje con Penrose hasta que disminuya la inflamación, así como el riesgo de obstrucción de la vía aérea. ${ }^{30}$

\section{Discusión}

La angina de Ludwig es una infección profunda de cabeza y cuello, potencialmente letal, que compromete de forma bilateral los espacios submandibular, sublingual y submentoniano. El diagnóstico de la angina de Ludwig está basado eminentemente en criterios clínicos, y debe ser realizado de manera oportuna para iniciar el tratamiento más adecuado y prevenir la aparición de complicaciones que pueden llegar a ser mortales. Las manifestaciones clínicas que debemos identificar, adicionalmente al compromiso bilateral de los espacios mencionados, son trismus, elevación de la lengua, disfagia y odinofagia. ${ }^{6,10}$ En algunos casos en los cuales hay un potencial compromiso de la vía aérea, el paciente puede adoptar la posición de olfateo ("sniffing"), presentar disfonía, estridor y utilizar los músculos accesorios de la respiración. ${ }^{3}$ Adicionalmente debemos identificar manifestaciones sistémicas del progreso del cuadro infeccioso como son fiebre, taquicardia y taquipnea.

Entre las potenciales complicaciones de la angina de Ludwig destacan edema de glotis, fascitis necrotizante y mediastinitis descendente necrotizante (la cual tiene la más elevada tasa de mortalidad). ${ }^{17,18} \mathrm{~A}$ pesar de la reducción en la incidencia y mortalidad de las complicaciones asociadas con la angina de Ludwig, debido al surgimiento y disponibilidad de nuevas terapias antibióticas durante los últimos ańos, muchos profesionales médicos y odontólogos desconocen sus complicaciones tempranas, las cuales pueden ser potencialmente mortales, en especial a nivel de la vía aérea, lo que puede retrasar el diagnóstico e imposibilitar el tratamiento oportuno. Por eso es sumamente importante reconocer las manifestaciones de la angina de Ludwig para poder instaurar rápidamente el tratamiento más adecuado.

Un dato importante a tomar en cuenta para elegir el tratamiento antibiótico más efectivo para los pacientes con diagnóstico de angina de Ludwig, es que se ha demostrado que más de dos terceras partes de las infecciones profundas de cabeza y cuello (entre las que se encuentra la angina de Ludwig) contienen bacterias productoras de betalactamasas. ${ }^{7}$ Por lo tanto, en localidades con altos niveles de resistencia bacteriana (como sucede en nuestro país debido al elevado índice de automedicación por parte de los pacientes y la utilización innecesaria de antibióticos en casos en los cuales no son requeridos) siempre será preferible elegir antibióticos resistentes a la acción de las betalactamasas, para asegurarnos de que el cuadro de angina de Ludwig será erradicado de forma más efectiva. Algunas excelentes alternativas para el tratamiento de la angina de Ludwig serían las asociaciones de Clindamicina con Amikacina, Ciprofloxacino con Metronidazol o Ampicilina/Sulbactam con Metronidazol. Un dato muy importante que debemos tener en cuenta es que el tratamiento antibiótico deberá prolongarse por lo menos durante 10 días y adecuarlo controlando estrictamente la evolución clínica del paciente.

\section{Conclusiones}

La angina de Ludwig es una celulitis que compromete bilateralmente los espacios submandibular, sublingual y submentoniano, y que generalmente se origina en infecciones odontogénicas de las molares mandibulares.

Si la angina de Ludwig no recibe el tratamiento adecuado puede progresar rápidamente a complicaciones potencialmente mortales como edema de glotis, fascitis necrotizante o mediastinitis.

El tratamiento de la angina de Ludwig consta de tres pilares fundamentales: el soporte de la vía aérea, el tratamiento farmacológico (antibióticos con actividad frente a anaerobios y resistentes a betalactamasas) y el tratamiento quirúrgico.

\section{Referencias bibliográficas}

1. Infante-Cossio P, Fernández-Hinojosa E, Mangas-CRZ M, González Pérez L. Ludwig's angina and ketoacidosis as a first manifestation of diabetes mellitus. Med Oral Pat Oral Cir Bucal 2010;15(4):e624e627. 
2. Jiménez Y, Bagán JV, Murillo J, Poveda $R$. Infecciones odontogénicas. Complicaciones. Manifestaciones sistémicas. Med Oral Patol Oral Cir Bucal 2004;9:S139-147.

3. Saifeldeen K, Evans R. Ludwig's angina. Emerg Med J 2004;21:242243.

4. Marcus B, Kaplan J, Collins K. A case of Ludwig angina: a case report and review of the literature. Am J Forensic Med Pathol 2008;29(3):255-9.

5. Kremer M, Blair T. Ludwig angina: forewarned is forearmed. AANA J 2006; 74(6):445-451.

6. Jiménez $S$, Murillo $G$, Britto $M$. Angina de Ludwig. Comunicación de un caso clínico. An Orl Mex 2008;53(3):129-33.

7. Boscolo-Rizzo P, Da Mosto MC. Submandibular space efection: a potentially lethal infection. International journal of infectious diseases 2009; 13: 327-333.

8. Manzo E, Méndez G, Hernández G, Salvatierra A, Vásquez M. Abscesos profundos de cuello. Etiopatogenia y morbimortalidad. Rev Asoc Mex Med Crit y Ter Int 2005;19(2):5459.

9. Leite A, Alves R, Dan S, Rocha A, Pereira C. Angina de Ludwig decorrente de exodontias de molares inferiores: relato de caso clínico. Rev Cir Traumatol Buco-Maxilo-Fac Camaragibe 2006;6(4):23-28.

10. Opeyemi O, Daramola M, Carrie E, Flanagan M, Robert H, Maisel $\mathrm{M}$, et al. Diagnosis and treatment of deep neck space abscesses. Otolaryngology-Head and Neck Surgery 2009;141(1):123-130.

11. Hueb MM, Borges LM, Oliveira LR. Angina de Ludwig: tratamento cirúrgico minimamente invasivo e guiado por ultrassonografia cervical. Revista Brasileira de Otorrinolaringologia 2004;8(3):279-86.

12. Ho MP, Tsai KC, Yen SL, Lu $\mathrm{CL}$, Chen $\mathrm{CH}$. A rare cause of Ludwig's angina by Morga- nella morganii. The Journal of Infection2006;53(4):e191-4.

13. Reynolds SC, Chow AW. Lifethreatening infections of the peripharyngeal and deep fascial spaces of the head and neck. Infect Dis Clin North Am 2007;21:557-76.

14. Lemos J, Caldeira J, Pereira T, Benez C, García I. Angina de Ludwig. Robrac. 2007;16(42):S1-9

15. Silva S, Rodrigues G, Alves M, Sirino P, Raimundo J, Farias M. Angina de Ludwig: revisão de literatura e relato de caso. Rev. Cir. Traumatol Buco-Maxilo-Fac Camaragibe 2009;9(3):9-14.

16. Hernández-Izquierdo D. Celulitis Facial Odontógena, su manejo como urgencia. Trabajo para optar el título de Máster en Urgencias Estomatológicas. Instituto Superior de Ciencias Médicas de la Habana. Facultad de Estomatología. Cuba 2008.

17. Vicente-Rodríguez JC. Celulitis maxilofaciales. Med Oral Patol Oral 2004;9:S126-38.

18. Gonzáles R, Vera M, Raffo M, Saldías R, Alarcón E, Gyhra A, et al. Mediastinitis necrotizante descendente. Revista Chilena de Cirugía 2005; 57(4):301-305.

19. Brommelstroet M, Fioravante J, Buffara P, Schmidlin C, Shibata $\mathrm{S}$. Mediastinitis Descendente Necrotizante pos Angina de Ludwig. Journal de Neumología. Sao Paulo 2001;27(5):269-271.

20. Morales, Nelda. "Evolución clínica de los pacientes con Angina de Ludwig sometidos a sedación consciente y a otras maniobras anestésicas". Tesis de Especialidad en Cirugia Bucomaxilo Facial. Universidad Nacional Autónoma de Nicaragua. 2003.

21. Chumpitaz V. Celulitis submandibular: caso clínico. Odontol Sanmarquina 2009;12(1):42-44.

22. Delgado M, Herrero Y, Daura A, Modelo A, Fernández E, Ruiz J et al. Eficacia de la monoterapia con piperacilina-tazobactam en infecciones del área maxilofacial. Rev Esp Cirug Oral y Maxilofac 2004;26:97-106.

23. Ocasio M, Martínez M, Cedeño A, Torres A, Alicea E, Rodríguez W. Ludwig's Angina: An Uncommon Cause of Chest Pain. Southern Medical Journal 2005; 98(5): 561-563.

24. Mehrotra, M; Mehrotra, S. Decompression of Ludwig Angina under Cervical Block. Anesthesiology 2002;97(3):1625-1626.

25. García-Villarmet C, De la TejaÁngeles E, Ceballos-Hernández $\mathrm{H}$, Ordaz-Favila J. Infecciones faciales odontogénicas. Informe de un caso. Acta Pediatr Mex 2007;28(3):95100.

26. García-Villarmet C, De la TejaÁngeles E, Ceballos-Hernández H, Ordaz-Favila J. Infecciones faciales odontogénicas. Informe de un caso. Revista Odontológica Mexicana 2009;13(3):177-183

27. Ngeow W. Ludwig's angina infection in a child: a cause for concern. Annal Dent Univ Malaya 1999;6:31-32.

28. Britt J, Josephson G, Gross C. Ludwig's angina in the pediatric population: report of a case and review of the literature. International Journal of Pediatric Otorhinolaryngology 2000;52:79-87.

29. Tejeda E, Rodríguez J, Gayosso O. Abscesos profundos de cuello. Reporte de cinco casos y revisión de la literatura. Rev Asoc Mex Med Crit y Ter Int 1999;13(4):142-145.

30. Callejas S, Cid A, Estebarán M. Drenaje de abscesos. Complejo Hospitalario de Toledo 2006;121:956. 